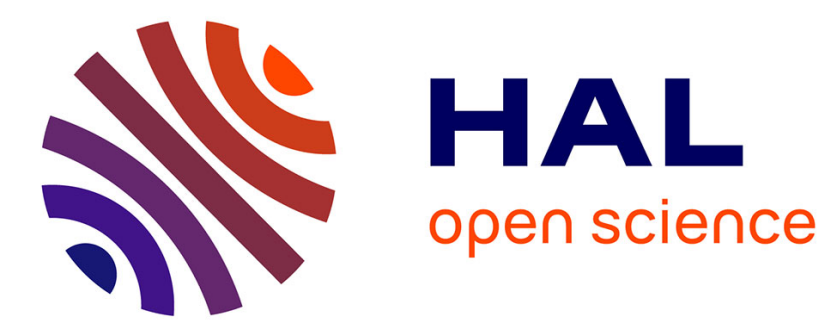

\title{
SPATIALLY VARYING BLUR RECOVERY. Diagonal Approximations in the Wavelet Domain
}

Paul Escande, Pierre Weiss, François Malgouyres

\section{To cite this version:}

Paul Escande, Pierre Weiss, François Malgouyres. SPATIALLY VARYING BLUR RECOVERY.

Diagonal Approximations in the Wavelet Domain. 2012. hal-00733194v2

\section{HAL Id: hal-00733194 \\ https://hal.science/hal-00733194v2}

Submitted on 28 Nov 2012

HAL is a multi-disciplinary open access archive for the deposit and dissemination of scientific research documents, whether they are published or not. The documents may come from teaching and research institutions in France or abroad, or from public or private research centers.
L'archive ouverte pluridisciplinaire HAL, est destinée au dépôt et à la diffusion de documents scientifiques de niveau recherche, publiés ou non, émanant des établissements d'enseignement et de recherche français ou étrangers, des laboratoires publics ou privés. 


\title{
SPATIALLY VARYING BLUR RECOVERY Diagonal Approximations in the Wavelet Domain
}

\author{
Paul Escande ${ }^{1}$, Pierre Weiss ${ }^{1}$ and François Malgouyres ${ }^{2}$ \\ ${ }^{1}$ ITAV-UMS3039, Université de Toulouse, CNRS, Toulouse, France. \\ 2 IMT-UMR5219, Université de Toulouse, CNRS, Toulouse, France. \\ paul.escande@gmail.com,pierre.armand.weiss@gmail.com,francois.malgouyres@math.univ-toulouse.fr
}

\begin{abstract}
Keywords: Image deblurring, spatially varying blur, operator approximation, wavelet packet transform, bi-harmonic spline interpolation, convex optimization.

Abstract: Restoration of images degraded by spatially varying blurs is an issue of increasing importance. Many new optical systems allow to know the system point spread function at some random locations, by using microscopic luminescent structures. Given a set of impulse responses, we propose a fast and efficient algorithm to reconstruct the blurring operator in the whole image domain. Our method consists in finding an approximation of the integral operator by operators diagonal in the wavelet domain. Interestingly, this method complexity scales linearly with the image size. It is thus applicable to large 3D problems. We show that this approach might outperform previously proposed strategies such as linear interpolations (Nagy and O'Leary, 1998) or separable approximations (Zhang et al., 2007). We provide various theoretical and numerical results in order to justify the proposed methods. We also show preliminary deblurring results illustrating the relevance of our formalism.
\end{abstract}

\section{INTRODUCTION}

Image restoration in the presence of spatially varying blur is a problem of increasing importance. It was first studied in the context of satellite imaging with Hubble space telescope (Nagy and O'Leary, 1998). It is now becoming increasingly important with the emergence of new fluorescence microscopes, producing highly deteriorated images, since light interacts with the biological tissues. In microscopy, it is often possible to incorporate micro-beads in the medium surrounding the sample or even in the sample itself, giving access to the point spread function (PSF) of the system at some known locations (see e.g. (Preibisch et al., 2010; Temerinac-Ott et al., 2011)). This information allows to interpolate the PSF in the whole space and thus to get approximations of the degradation operator for further processing.

In the case of spatially invariant blur, fast deconvolution algorithms can be devised since the convolution is diagonal in the Fourier domain. This allows using $O\left(d n^{d} \log (n)\right)$ algorithms (where $d$ denotes the space dimension and $n^{d}$ denotes the number of pixels) based on the fast Fourier transform. These approaches are unsuitable in the case of spatially varying blurs and it appeals for the development of new fast numerical algorithms. Our aim in this paper is to propose fast $O\left(n^{d}\right)$ algorithms based on the wavelet or wavelet packet transforms.

We consider a blurring operator $H$ in $\mathbb{R}^{d}$ and defined for any $u \in L^{2}(\Omega)$ as the following integral operator :

$$
\forall x \in \Omega,(H u)(x):=\int_{y \in \Omega} K(x, y) u(y) d y,
$$

where $\Omega \subseteq \mathbb{R}^{d}$ is the image domain. The function $K(x, \cdot)$ is a spatially varying kernel defining the PSF at each location $x$. In all the following, we assume that $H$ is a bounded linear operator from $L^{2}(\Omega)$ to $L^{2}(\Omega)$. The most naive approach to compute $H u$ numerically consists in discretizing (1) by :

$$
\forall x \in X, H u(x)=\sum_{y \in X} K(x, y) u(y),
$$

where $X \subset \Omega$ denotes the set of pixels locations. This approach is simple to implement, but costs $O\left(n^{2 d}\right)$ arithmetic operations. This is unsuitable for large $2 D$ images or medium sized $3 D$ images. Two alternative approaches are commonly used :

- The first one consists in approximating $K(x, \cdot)$ by a tensor product of kind :

$$
K\left(x,\left(y_{1}, \cdots, y_{d}\right)\right)=\prod_{k=1}^{d} K_{k}\left(x, y_{k}\right) .
$$



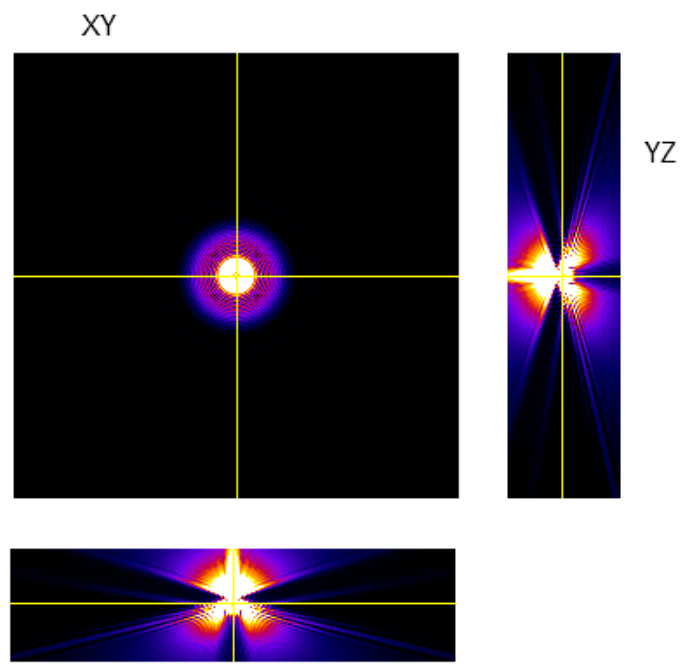

$X Z$

FIGURE 1 - An orthogonal view of a Variable Refractive Gibson and Lanni PSF obtained with the PSF Generator plugin for ImageJ (Kirshner et al., 2011)

This reduces the computational cost to $O\left(d n^{d+1}\right)$ operations which is usually tractable even in large scale scenarii. Moreover this model is exact for Gaussian PSF which sometimes accurately describe perfect microscopy systems (Zhang et al., 2007). Unfortunately, it is too rough to describe more complex patterns commonly encountered in optical diffraction or sample induced degradations. Figure 1 shows a typical PSF in three dimensions, which cannot be approximated by separable functions.

- The second one consists in using piecewise constant blurs using local FFT (Nagy and O'Leary, 1998; Hansen et al., 2006). Its complexity is roughly the same as that of spatially invariant blurs in $O\left(d n^{d} \log (n)\right)$. Moreover, this approach also allows to use linear interpolations of the PSF. This might be interesting in our case since the PSF is known only at a few positions and linear interpolations allow an operator reconstruction on the whole image domain. Unfortunately, piecewise constant blurs or linear interpolations are too rough to describe some practical settings. This is illustrated on Figure 9(b), where we can observe that the linear interpolation gives a cross like PSF in the middle, that would be undesirable in practical cases.

In this work, we propose to approximate $H$ by operators diagonal in wavelet or wavelet packet transforms. More precisely, we show that $H \simeq \Psi \Sigma \Psi^{*}$, where $\Psi$ denotes the wavelet transform and $\Sigma$ is a diagonal matrix. The computation of $\mathrm{Hu}$ is thus reduced to $O\left(n^{d}\right)$ operations.

The structure of the paper is as follows. In section 3 , we justify the use of such a structure by theoretical and numerical results. In section 4 , we propose an algorithm to reconstruct the diagonal operator $\Sigma$ when the impulse response of $H$ is given at some known locations. Finally, we present a deconvolution algorithm and some results in section 5 .

\section{NOTATION}

In order to simplify the notation, we consider wavelet transforms and not wavelet packet transforms. We present the theoretical results in 1D for the sake of simplicity and clarity and the experimental results in 2D. The proposed approaches can be extended to any dimensions and would be particularly suited to large 3D problems. $\mathbb{L}^{2}(\mathbb{R})$ :

We consider an orthogonal wavelet basis of

$$
\left\{\phi_{l_{0}, n}\right\}_{n \in \mathbb{Z}} \cup\left\{\psi_{j, n}\right\}_{j \leq l_{0}, n \in \mathbb{Z}}
$$

where

$$
\psi_{j, n}(t)=\sqrt{2^{-j}} \psi\left(2^{-j} t-n\right),
$$

and $\psi$ is the mother wavelet. The function $\phi_{l_{0}, n}$ is defined by

$$
\phi_{l_{0}, n}(t)=\sqrt{2^{-l_{0}}} \phi\left(2^{-l_{0}} t-n\right),
$$

where $\phi$ is the scaling function.

In all the paper, $\Psi^{*}$ denotes the forward wavelet transform and $\Psi$ denotes its inverse (in the discrete and in the continuous setting). $\mathcal{F}^{*}$ denotes the Fourier transform (discrete or continuous) and $\mathcal{F}$ denotes its inverse. The convolution between $u$ and $h$ is denoted $h \star u$. The Fourier transform of $u$ is denoted $\hat{u}$ or $\mathcal{F}^{*} u$

The indicator function of a convex and closed set $C \subseteq \mathbb{R}^{n}$ is denoted $\chi$ and defined as :

$$
\chi_{C}(x)= \begin{cases}0 & \text { if } x \in C \\ +\infty & \text { otherwise. }\end{cases}
$$

The proximal operator or resolvent of a convex, closed function $F: \mathbb{R}^{n} \rightarrow \mathbb{R} \cup\{+\infty\}$ is defined for all $x_{0} \in \mathbb{R}^{n}$ by :

$$
\left(I+\partial F^{*}\right)^{-1}\left(x_{0}\right)=\underset{x \in \mathbb{R}^{n}}{\arg \min } F(x)+\frac{1}{2}\left\|x-x_{0}\right\|_{2}^{2} .
$$

For a discrete image in $\mathbb{R}^{d}$, we define the discrete partial derivative in direction $i$ by :

$\partial_{i} u(\cdot, k, \cdot)= \begin{cases}u(\cdot, k+1, \cdot)-u(\cdot, k, \cdot) & \text { if } 1 \leq k<n \\ 0 & \text { if } k=n .\end{cases}$ 
where the indice $k$ is that the $i$-th position in the array. The discrete gradient operator in $\mathbb{R}^{d}$ is defined by :

$$
\nabla=\left(\begin{array}{c}
\partial_{1} \\
\partial_{2} \\
\vdots \\
\partial_{d}
\end{array}\right)
$$

Let $q \in\left(\mathbb{R}^{n^{d}}\right)^{d}$ represent a discrete vector field. We set

$$
q=\left(\begin{array}{c}
q_{1} \\
q_{2} \\
\vdots \\
q_{d}
\end{array}\right) .
$$

The isotropic $l^{1}$-norm in $\left(\mathbb{R}^{n^{d}}\right)^{d}$ is defined by :

$$
\|q\|_{1,2}=\sum_{i=1}^{n^{d}} \sqrt{\sum_{j=1}^{d} q_{j}(i)^{2}}
$$

Finally, the discrete total variation of $u \in \mathbb{R}^{n^{d}}$ is defined by :

$$
T V(u)=\|\nabla u\|_{1,2}
$$

\section{DIAGONALIZATION OF THE VARIABLE BLUR OPERATOR IN A WAVELET BASIS}

The main ingredient allowing the design of efficient deconvolution algorithms is the fact that a convolution is diagonalized in the Fourier domain. For any kernel $h, h \star u=\mathcal{F} \Sigma \mathcal{F}^{*} u$ where $\Sigma$ can be considered as a diagonal operator that multiplies $\mathcal{F}^{*} u$ by $\mathcal{F}^{*} h$. The main idea of this paper is to mimic this property for spatially varying blur operators. We propose to approximate $H$ by an operator $\tilde{H}$ diagonal in the wavelet domain :

$$
\begin{aligned}
H u & \simeq \tilde{H} u \\
& :=\Psi \Sigma \Psi^{*} u \\
& =\sum_{n \in \mathbb{Z}}\left\langle\phi_{l_{0}, n}, u\right\rangle \phi_{l_{0}, n}+\sum_{j \leq l_{0}, n \in \mathbb{Z}} \sigma_{j, n}\left\langle\psi_{j, n}, u\right\rangle \psi_{j, n}
\end{aligned}
$$

where $\left(\sigma_{j, n}\right)_{j, n}$ is a sequence of weights that will be described later. This particular structure allows to approximate $H u$ in $O\left(n^{d}\right)$ arithmetic operations, which is doable even for very large scale problems.

Such operators have been deeply analyzed from a theoretical point of view in various articles or monographs (see e.g. (Beylkin et al., 1991; Coifman and Meyer, 1997)). However, we found very few image processing applications in the literature. To our knowledge, the closest practical application is dedicated to the fast computation of image foveation (Chang et al., 1999). However, this work is only adapted to very particular kind of kernels $K$ met in foveation that do not correspond to our practical problems.

Since $H$ is a linear operator in a Hilbert space, it can be written as :

$$
H=\Psi \Theta \Psi^{*},
$$

where $\Theta: l^{2} \rightarrow l^{2}$ is characterized by the coefficients,

$$
\left(\theta_{j, m, k, n}\right)_{j, m, k, n}:=\left(\left\langle H \psi_{j, m}, \psi_{k, n}\right\rangle\right)_{j, m, k, n} .
$$

In order to justify the proposed approach, we first recall some theoretical results presented in (Beylkin et al., 1991) that assess the decrease of $\theta_{j, m, k, n}$ away from the diagonal (i.e. when $|m-n|>0$ and $|j-k|>$ $0)$. Then we provide an interpretation of the coefficients $\sigma_{j, n}$ in terms of amplitudes of the Fourier coefficients of the local PSF.

\subsection{Decay of $\Theta$ Away from the Diagonal}

In (Beylkin et al., 1991), it has been proved that, for compactly supported wavelets possessing $M$ vanishing moments and smoothly varying kernels, the values of $\Theta$ are small away from the diagonal in the one and two-dimensional cases. Typical results are as follow :

Theorem 1 ((Beylkin et al., 1991)). Suppose that $|K(x, y)| \leq \frac{1}{|x-y|}$ and that $K(x, y)$ is of class $C^{M+1}$ with

$$
\left|\partial_{x}^{M} K(x, y)+\partial_{y}^{M} K(x, y)\right| \leq \frac{C_{M}}{|x-y|^{(1+M)}},
$$

where $M$ denotes the number of vanishing moments of $\psi$. Then $\theta_{j, m, k, n}$ satisfies the following inequality:

$$
\left|\theta_{j, m, k, n}\right| \leq O\left(\frac{1}{1+|j-k|^{M+1}}\right) .
$$

Moreover, for compactly supported kernels $K$ :

$$
\left|\theta_{j, m, k, n}\right|=0,
$$

for sufficiently large $|m-n|$.

The authors also show that the operator norm $\left\|H-\Psi \tilde{\Theta} \Psi^{*}\right\|$ can be made arbitrarily small if $\tilde{\Theta}$ is obtained by thresholding $\Theta$ in such a way that only $O\left(n^{d}\right)$ coefficients are kept. It roughly means that if $K$ is a smooth kernel, computing $\mathrm{Hu}$ can be performed in $O\left(n^{d}\right)$ operations, rather than $O\left(n^{2 d}\right)$, by making use of the wavelet transform. In this work, rather than considering sparse matrices $\tilde{\Theta}$, we use simpler diagonal matrices.

We illustrate these results experimentally in the discrete setting on Figure 3. We consider an operator $H$ whose kernel is a two-dimensional Gaussian with 
variances linearly increasing in the vertical direction, see Figure 2(c). This operator applied to the mandrill image results in the image Figure 2(b). The matrix $\Theta$ is shown on Figure 3. It is seen that $\Theta$ is dominated by its diagonal entries and that the coefficients away from the diagonal decrease extremely fast (actually much faster than the result in Theorem 1).

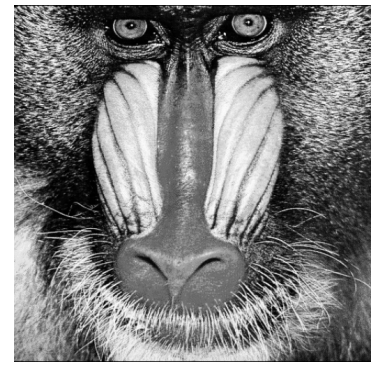

(a) Original Image

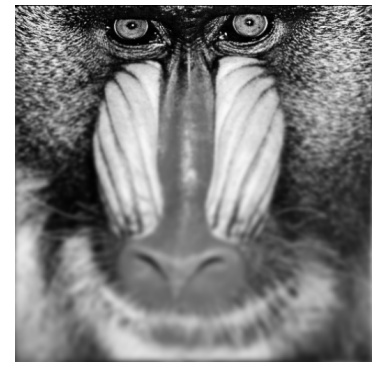

(b) Blurred Image

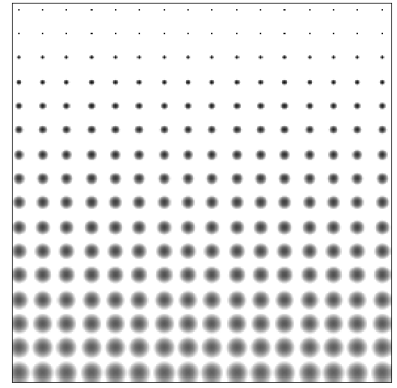

(c) PSF at various locations

FIGURE 2 - Image blurred using the operator $H$. The kernel $K$ of the operator is a Gaussian which grows linearly in the vertical direction.

\subsection{Interpretation of the Diagonal Values}

In this paragraph, we show that the values $\sigma_{j, n}$ can be interpreted as local frequency responses of $\tilde{H}$. We assume that $\psi$ is a compactly supported wavelet on the interval $[-\beta, \beta]$.

Let us analyze the impulse response of $\tilde{H}$ at point $x$ :

$$
\begin{aligned}
\tilde{H} \delta_{x} & =\Psi \Sigma \Psi^{*} \delta_{x} \\
& =\sum_{n \in \mathbb{Z}} \phi_{l_{0}, n}(x) \phi_{l_{0}, n}+\sum_{j \leq l_{0}, n \in \mathbb{Z}} \sigma_{j, n} \psi_{j, n}(x) \psi_{j, n} \\
& =\sum_{n \in \mathbb{Z}} \phi_{l_{0}, n}(x) \phi_{l_{0}, n}+\sum_{\substack{j \leq l_{0}, n \in k(x, j)}} \sigma_{j, n} \psi_{j, n}(x) \psi_{j, n},
\end{aligned}
$$

where

$$
k(x, j):=\left\{n \in \mathbb{Z} \text { such that }\left|2^{-j} x-n\right|<\beta\right\} .
$$

The sets $k(x, j)$ are represented in Figure 4 in the two-dimensional case. They contain at most $\lfloor 2 \beta\rfloor$ elements.

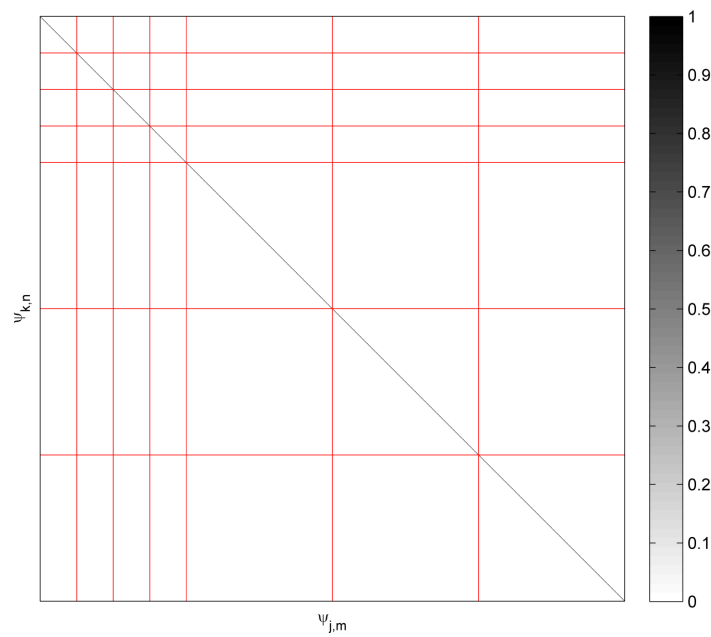

(a) In a linear scale

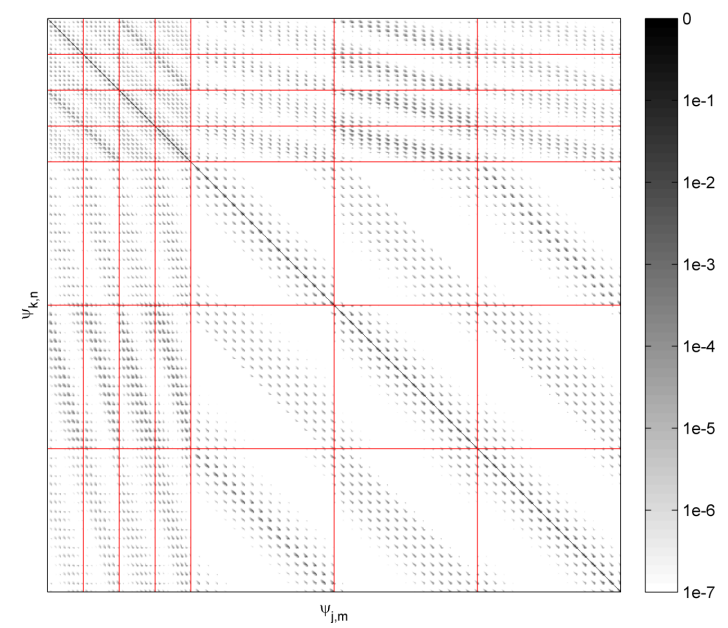

(b) In a $\log _{10}$ scale

FIGURE 3 - Matrix $\Theta$ for the variable operator illustrated in Figure 2. This matrix is obtained using Daubechies 8 wavelets and a decomposition level $\mathrm{J}=2$.

Now, if we assume that $\sigma_{j, n}$ varies little in $k(x, j)$ and satisfies $\sigma_{j, n} \simeq \sigma_{j, x}$ we obtain :

$$
\begin{aligned}
\Psi \Sigma \Psi^{T} \delta_{x} & \simeq \sum_{n \in k\left(x, l_{0}\right)} \phi_{l_{0}, n}(x) \phi_{l_{0}, n} \\
& +\sum_{j \leq l_{0}} \sigma_{j, x}\left(\sum_{n \in k(x, j)} \psi_{j, n}(x) \psi_{j, n}\right) .
\end{aligned}
$$

The local frequency response of $\tilde{H}$ is thus

$$
\begin{aligned}
& \widehat{\Psi \Sigma \Psi^{*} \delta_{x}} \\
& \simeq \sum_{n \in \mathbb{Z}} \phi_{l_{0}, n}(x) \widehat{\phi_{l_{0}, n}}+\sum_{j \leq l_{0}} \sigma_{j, x}\left(\sum_{n \in k(x, j)} \psi_{j, n}(x) \widehat{\psi_{j, n}}\right) \\
& =\sum_{n \in \mathbb{Z}} \phi_{l_{0}, n}(x) \widehat{\phi_{l_{0}, n}}+\sum_{j \leq l_{0}} \sigma_{j, x} \alpha_{j, x} \widehat{\psi_{j}},
\end{aligned}
$$




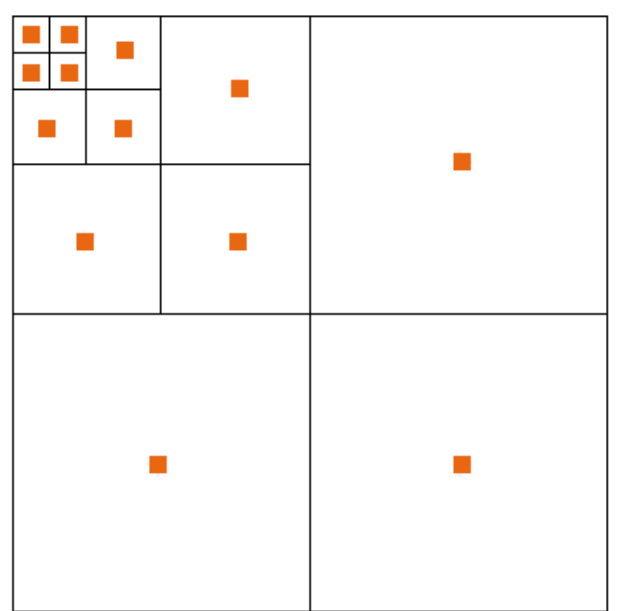

FIGURE 4 - The sets $k(x, j)$ are indicated in orange at each scale.

where $\alpha_{j, x}$ is a complex coefficient that depends on the choice of $\psi$ and $\psi_{j}(x)=\sqrt{2^{-j}} \psi\left(2^{-j} x\right)$. Since $\widehat{\psi_{j}}$ is well localized in the frequency domain, the coefficient $\sigma_{j, x} \alpha_{j, x}$ can be interpreted as a local frequency attenuation in a certain frequency band that depends solely on the scale $j$. This principle is illustrated in Figure 5.

\subsection{Spatial Regularity of the Eigenvalues}

A simple way to find a matrix $\Sigma$ such that $\tilde{H} \simeq H$ consists in setting $\Sigma=\operatorname{Diag}(\Theta)$. If the kernel $K$ varies sufficiently smoothly in space, the discrete values $\left(\sigma_{j, n}\right)_{n \in \mathbb{Z}}$ also vary smoothly, meaning that $\sigma_{j, n} \simeq$ $\sigma_{j, n+1}$. This can be verified experimentally : Figure 6 represents the diagonal of $\Theta$ for an operator $H$ displayed in Figure 2(c) in the usual wavelet domain. The eigenvalues vary smoothly in each sub-band. This remark is central to understand the interpolation algorithm proposed in the next section.

Also notice that the coefficients $\sigma_{j, n}$ decrease from the top to the bottom of the image at each scale. It means that the high-frequencies are attenuated on the image bottom. This clearly corresponds to the operator $H$ shown in Figure 2(c).

\subsection{Wavelet and Basis Choice}

An important parameter in the proposed algorithm is the mother wavelet $\psi$. According to Theorem 1, $\psi$ should have many vanishing moments for $\tilde{H}$ to approximate $H$ correctly. This condition can be verified experimentally. In the following, we will always consider Daubechies 8 orthogonal wavelet bases

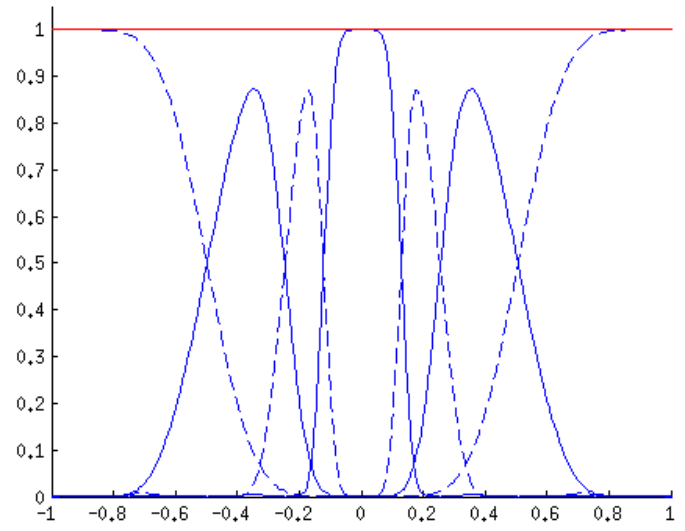

(a) In blue $\left|\widehat{\psi_{j}}\right|^{2}$ and in red $\sum_{j}\left|\widehat{\psi_{j}}\right|^{2}$

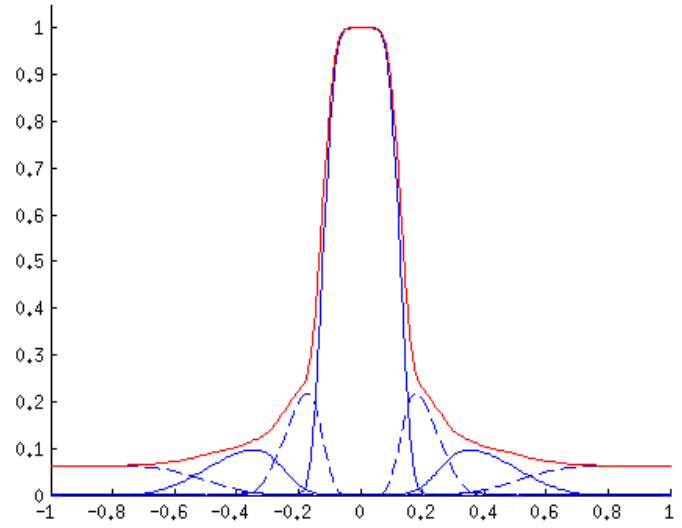

(b) In blue $\left|\sigma_{j, x} \alpha_{j, x}\right| \widehat{\psi_{j}}||$ and in red $\left|\sum_{j} \sigma_{j, x} \alpha_{j, x} \widehat{\psi_{j}}\right|$

FIGURE 5 - Local Fourier attenuation are determined by the coefficients $\sigma_{j, x} \alpha_{j, x}$.

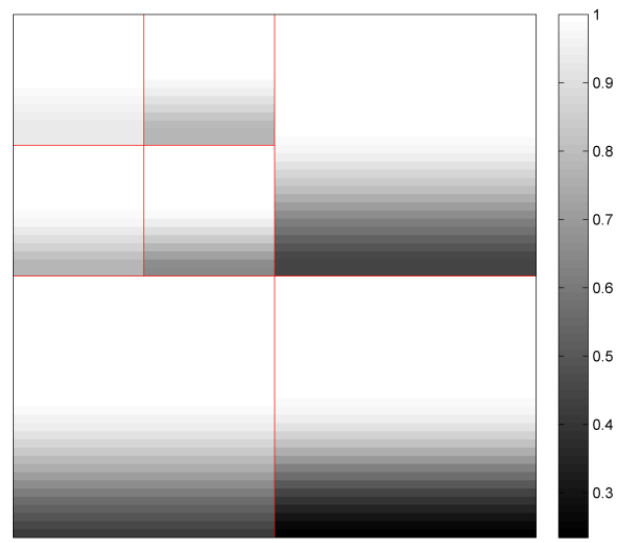

FIGURE 6 - Diagonal of the matrix $\Theta$.

which appeared to produce good practical results.

It was shown in (Malgouyres, 2002; Kalifa et al., 2003), that wavelet packet bases are more adapted to deconvolution problems than standard wavelet bases. The reason is that they provide more precise fre- 
quency tilings, notably in the high frequencies. This is illustrated in Figure 7. The frequency tiling of the standard wavelet decomposition is much coarser than that of a wavelet packet transform.

Finally, the non translation invariance of discrete wavelets tend to produce ringing artifacts. They can be reduced using redundant bases such as the translation invariant wavelets. In this work we use cycle spinning (Coifman and Donoho, 1995), which consists in averaging wavelet transforms translated in the set $\{0,1\}^{d}$. This transform is a tight frame with a redundancy of $2^{d}$.

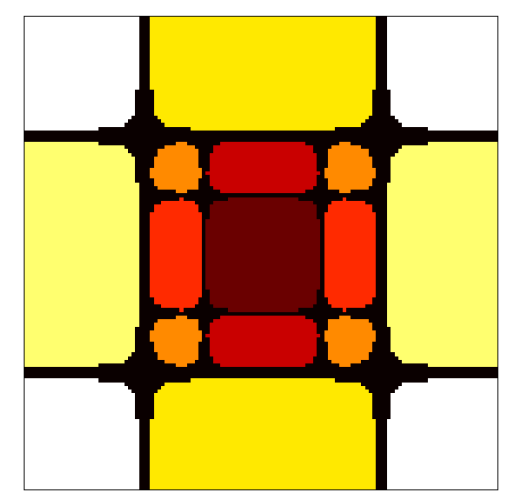

(a) In a wavelet wasis

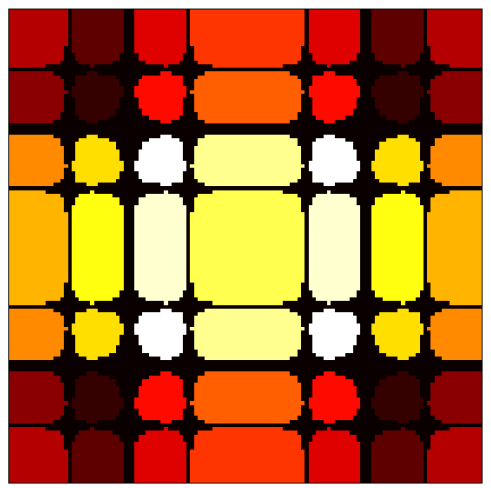

(b) In a wavelet packet basis

FIGURE 7 - Frequency tilings for Daubechies-8 wavelets in the wavelet and wavelet packet basis. Each color corresponds to the Fourier transform of a wavelet at a given scale.

\section{OPERATOR RECONSTRUCTION FROM LOCALLY KNOWN PSF}

In this section, we propose a method to recover the matrix $\Sigma$ from the knowledge of local impulse responses. This setting corresponds to various practical applications. In astronomy, stars may sometimes be considered as Diracs. Their observation thus provides the impulse response of the system $K(x, \cdot)$, where $x$ denotes the star location. In microscopy, micro-beads may be inserted in the sample and provide the impulse responses at locations spread in the whole image domain.

The problem tackled in this section is the reconstruction of $K$ everywhere, from the knowledge of $K\left(x_{i}, \cdot\right)$ at a few locations $\left(x_{i}\right)_{i \in\{1, \cdots, m\}}$. We assume that two images are available :

- An image

$$
u=\sum_{i=1}^{m} \delta_{x_{i}},
$$

that describes the Dirac locations.

- An image $u_{o}=H u$ which provides the impulse responses at locations $x_{i}$.

Figure 8 illustrates two images $u$ and $u_{o}$. The Diracs could be randomly located on the image rather than on a uniform grid. We considered this simple setting for experimental reasons. The number of known impulse responses can also be considerably reduced as will be shown later.

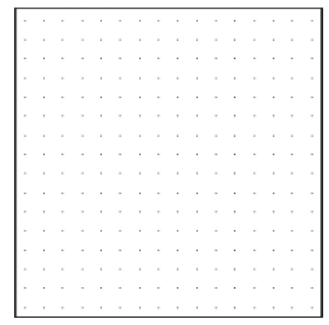

(a) The dirac map $u$

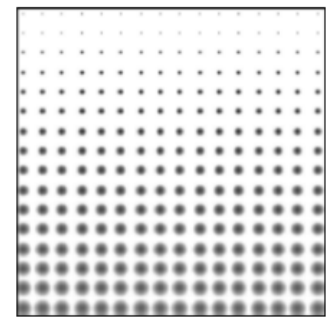

(b) $u_{o}=H u$
FIGURE 8 - Dirac map and the associated impulse responses. This information is used to reconstruct an approximation of the blurring operator $H$.

The knowledge of $u_{o}$ allows to reconstruct the eigenvalues $\sigma_{j, n}$ of $\tilde{H}$ only close to the known locations $x_{i}$ in each sub-band. These eigenvalues should thus be interpolated in order to recover $K$ everywhere. Note that this problem is not standard since is consists in interpolating an operator eigenvalues and not an image.

Since the eigenvalues vary smoothly in space, we propose to use bi-harmonic splines which are well adapted to scattered data interpolation (Wahba, 1990). The approximation problem we propose formulates as the following variational problem :

Find $\Sigma \in \arg \min \left\|\Psi \Sigma \Psi^{*} u-u_{o}\right\|_{2}^{2}+\lambda R(\Sigma)$, where $\lambda>0$ is a regularization parameter. We also set :

$$
R(\Sigma)=\sum_{j \leq l_{0}}\left\|\Delta \sigma_{j}\right\|_{2}^{2},
$$

where $\Delta$ denotes the discrete Laplacian and $\sigma_{j}$ denotes the set of eigenvalues at scale $j$. This energy 
provides the approximation of minimal curvature. It is equivalent to using bi-harmonic splines (Wahba, 1990).

The quadratic structure of problem (3) allows the use of conjugate gradient like methods for the minimization. We are currently investigating the use of preconditionners in the wavelet domain for accelerating the convergence.

We present approximation results in Figures 9 and 10. Figure 10 displays a interpolated matrix $\Sigma$. This result can be evaluated by comparing it with the true diagonal of $\Theta$ presented in Figure 6. Overall, the reconstruction leads to near perfect results. Figure 9 compares the interpolation provided by Fourier based methods such as (Nagy and O'Leary, 1998) with the proposed approach. Our method produces some artifacts, however, the proposed interpolation is rather close to the reality in the image center. Note that this result is obtained using knowing the PSF at only 4 locations in the plane. Deblurring an image with kernel 9(b) would be disastrous, since horizontal and vertical frequencies would be enhanced, leading to strong ringing artifacts.

\section{DEBLURRING ALGORITHM AND RESULTS}

\subsection{Deblurring Method}

In this section, we assume that the diagonal $\Sigma$ has been reconstructed using the method proposed in section 4 . We propose a total variation (TV) based algorithm to tackle the deblurring problem. We suppose that a degraded image $v_{o}$ is obtained according to the following discrete model :

$$
v_{o}=H v+\eta,
$$

where $H: \mathbb{R}^{n^{d}} \rightarrow \mathbb{R}^{n^{d}}$ is the spatially varying blur operator, $v \in \mathbb{R}^{n^{d}}$ is the unaltered image and $\eta \in \mathbb{R}^{n^{d}}$ is a white Gaussian noise, $\eta \sim \mathcal{N}\left(0, \sigma_{\eta} I d_{n^{d}}\right)$. Our aim is to recover $v$ knowing $v_{0}$. Since $\tilde{H}$ and $H$ are usually compact, the inverse problem of recovering $v$ should be regularized. We propose to use a standard total variation based reconstruction approach. It reads :

$$
\text { Find } \underset{v \in \mathbb{R}^{n^{d}},\left\|\tilde{H} v-v_{o}\right\|_{2}^{2} \leq \alpha}{\arg \min } T V(v)
$$

where $\alpha>0$ is a user fixed parameter and $T V(v)$ is the isotropic total variation of $v$ defined in the notation. In settings where $H$ is perfectly known, users should set $\alpha=\sigma^{2} n$. The proposed approach slightly differs

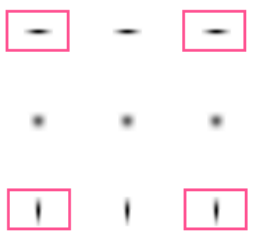

(a) Exact PSF

(b) Linearly Interpolated PSF

(c) Our Interpolated PSF

FIGURE 9 - Operator reconstruction using different methods. The operator is reconstructed using the information available in the red rectangles.

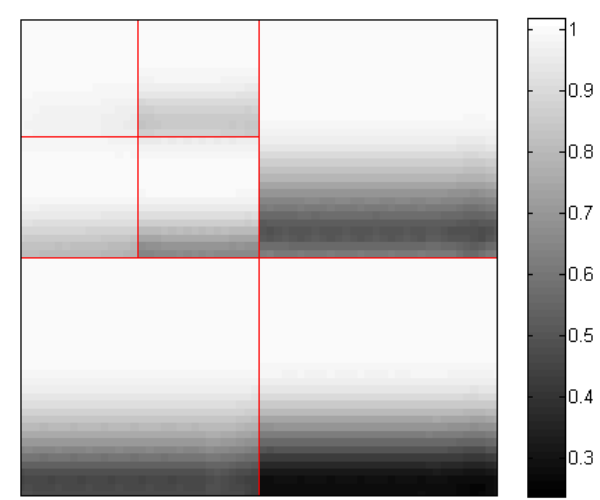

FIGURE 10 - The matrix $\Sigma$ reconstructed using bi-harmonic splines. It should be compared to the real diagonal presented on Figure 6.

since total variation serves as a regularizer for both the noise and the errors in the operator approximation. In 
practice we found that setting $\alpha=(1+\varepsilon) \sigma^{2} n$ where $\varepsilon>0$ is a small parameter provides good experimental results.

Problem (4) can be rewritten as :

$$
\min _{v \in \mathbb{R}^{n^{d}}} F(A v)+G(v)
$$

where

$$
\begin{aligned}
& A: \quad \mathbb{R}^{n^{d}} \rightarrow \mathbb{R}^{d n^{d}} \times \mathbb{R}^{n^{d}} \\
& v \mapsto\left(\begin{array}{c}
\nabla v \\
\tilde{H} v
\end{array}\right), \\
& F: \quad \mathbb{R}^{d n^{d}} \times \mathbb{R}^{n^{d}} \rightarrow \mathbb{R}_{+} \\
& \left(y_{1}, y_{2}\right) \quad \mapsto\left\|y_{1}\right\|_{1,2} \\
& +X_{\left\{y,\left\|y_{2}-v_{o}\right\|_{2} \leq \alpha\right\}}\left(y_{2}\right)
\end{aligned}
$$

and

$$
G=0 .
$$

This reformulation allows to use the primal-dual algorithm proposed in 1 detailed in algorithm 1 .

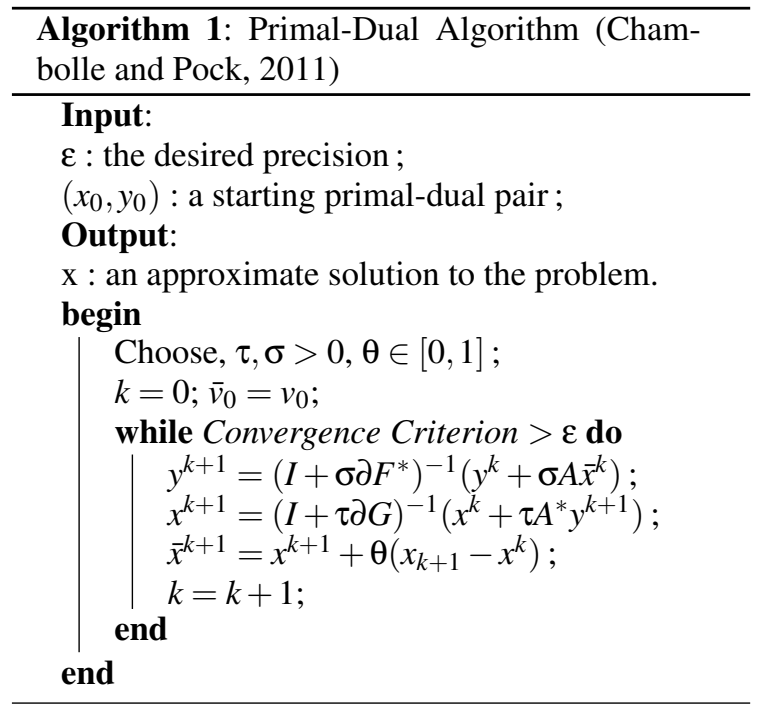

This algorithm is easy to implement and it can be shown to converge in $O\left(\frac{1}{k}\right)$. This rate is somehow optimal in the class of first order methods (Nemirovskii and Yudin, 1979). The proximal operators of $F^{*}$ and $G$ are easy to compute analytically and we refer the reader to (Combettes et al., 2006; Chambolle and Pock, 2011) for more details. The steps sizes $\tau$ and $\sigma$ should satisfy $\sigma \tau\|A\|^{2}<1$ for convergence, where $\|A\|=\max _{j, n}\left|\sigma_{j, n}\right|$. In practice, this algorithm requires around 100 low cost iterations to provide satisfactory results for the visual system. Note that $\tilde{H}$ and $\tilde{H}$ must be applied to a vector at each iteration. This is performed in $O\left(n^{d}\right)$ arithmetic operations due to the special structure of $\tilde{H}$.

\subsection{Results for Different Noise Variances}

Now we will present some results of the deblurring algorithm.

We used the Mandrill Figure 11(a) rescaled in $[0,1]$ and blurred with an operator having a twodimensional Gaussian PSF with variance increasing linearly in the vertical direction. It impulse responses are displayed in 11(b). In Figures 12 and 13 we respectively added a noise of variance $\sigma_{\eta}=0$ and $\sigma_{\eta}=$ $3.10^{-2}$.

In the case $\sigma_{\eta}=0$, Figure 12 shows that the algorithm is able to recover thin details of the image even in the coat and the beard of the Mandrill in the bottom of the image. This highlights the fact that the approximation of $H$ by $\tilde{H}$ is sufficiently good for the sake of deblurring.

In the case of a larger noise, $\sigma_{\eta}=3.10^{-2}$, Figure 13 shows that the image quality is improved but suffers from the standard defects of total-variation based regularizations : stair-case appears and thin details are not recovered. Overall, these results confirm that the proposed approximation is capable of producing nice reconstruction results with low computational costs.

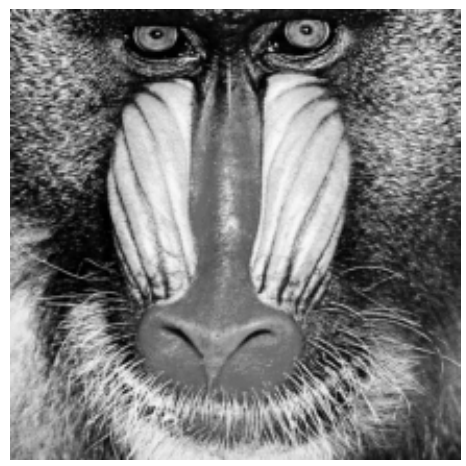

(a) Original Image

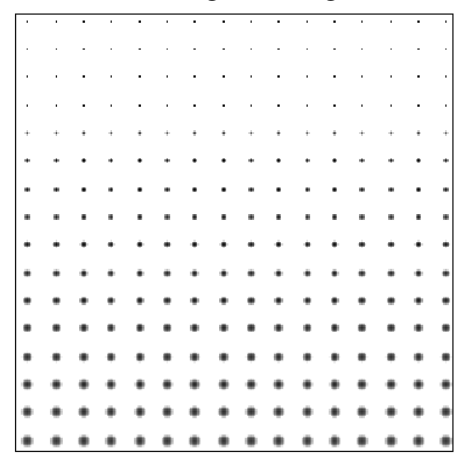

(b) The PSF

FIGURE 11 - The original Image and the impulse response of the blurring operator. 


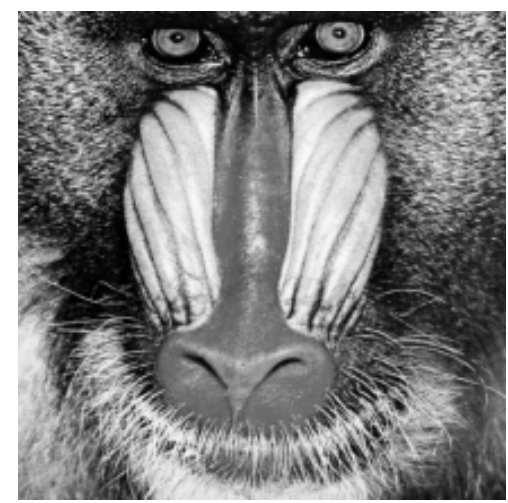

(a) Original Image

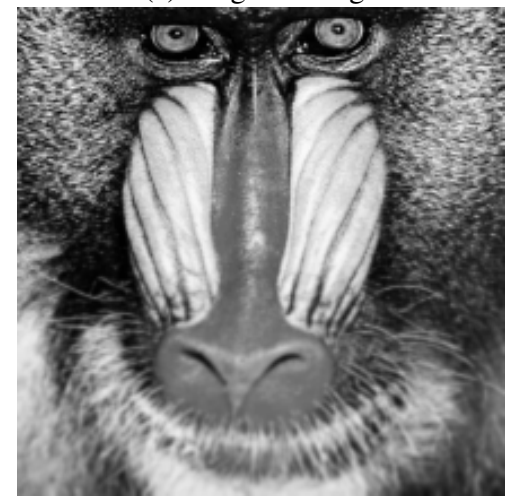

(b) Degraded Image $S N R=22.51$

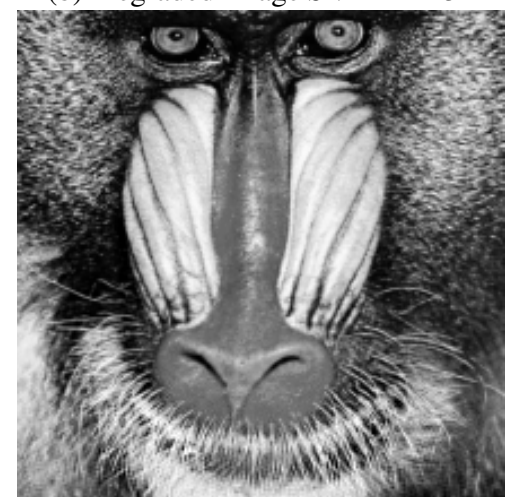

(c) Deblurred Image, $S N R=29.02$

FIGURE 12 - Restoration results for $\sigma_{\eta}=0$.

\section{CONCLUSION}

This paper contains various contributions. First, we showed that spatially varying blur operators can be well approximated by operators diagonal in the wavelet domain. Second, we proposed an original approach that allows to reconstruct blur operators when their PSF is known only at a few discrete locations. Compared to previously proposed approaches, this method allows a much wider class of interpolation algorithms. In particular, we showed that bi-harmonic

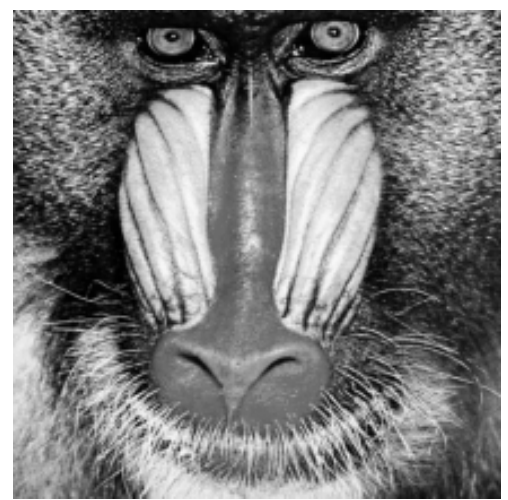

(a) Original Image

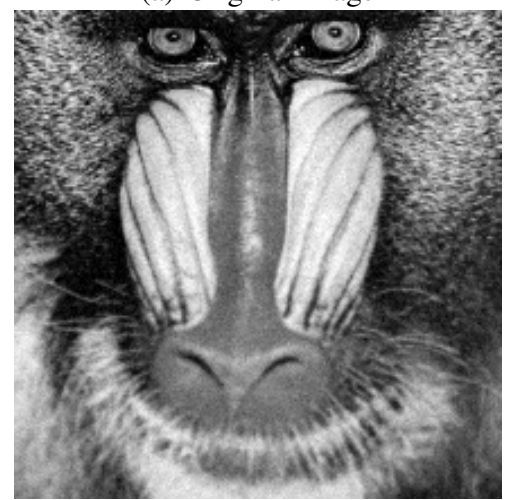

(b) Degraded Image $S N R=20.58$

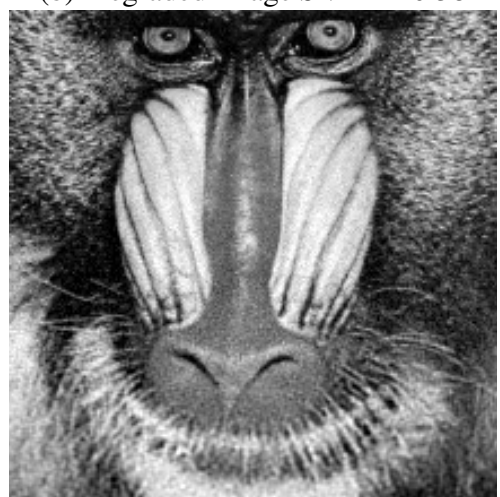

(c) Deblurred Image, $S N R=20.86$

FIGURE 13 - Restoration results for $\sigma_{\eta}=3 \cdot 10^{-2}$.

splines interpolations produce near perfect results on a few examples and outperform standard approaches. Finally we presented preliminary deblurring results. These results outline that the proposed approximations provide results similar to what would be obtained with a perfect knowledge of the blur operator.

The proposed algorithm will be validated on real data coming from emergent microscopes such as the selective plane illumination microscope. 


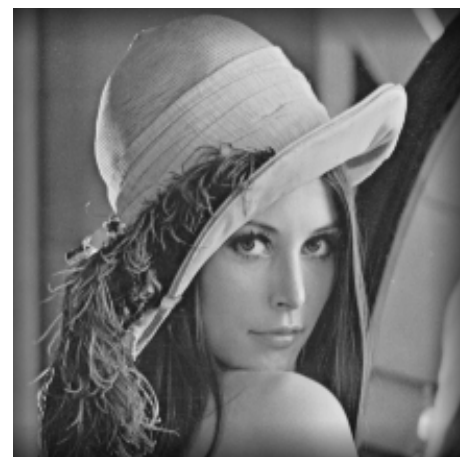

(a) Original Image

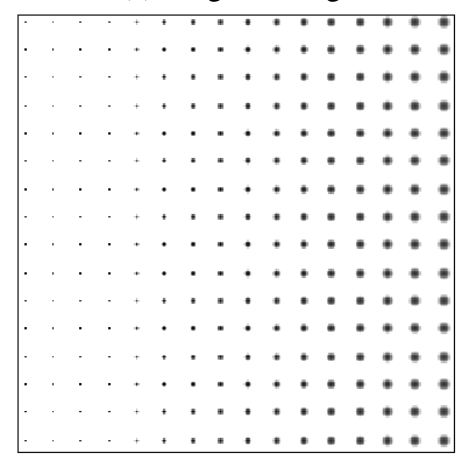

(b) The PSF

FIGURE 14 - The original image and the and the impulse response of the blurring operator.

\section{ACKNOWLEDGEMENTS}

This work was partially funded by the Mission pour l'interdisciplinarité from CNRS, Région Midi Pyrénées and the ANR SPHIM3D.

The authors wish to thank J. Bigot and J. Fehrenbach for interesting discussions. They also thank all the ITAV staff, Charlotte Emery and Emmanuel Soubies for their tireless support during this project.

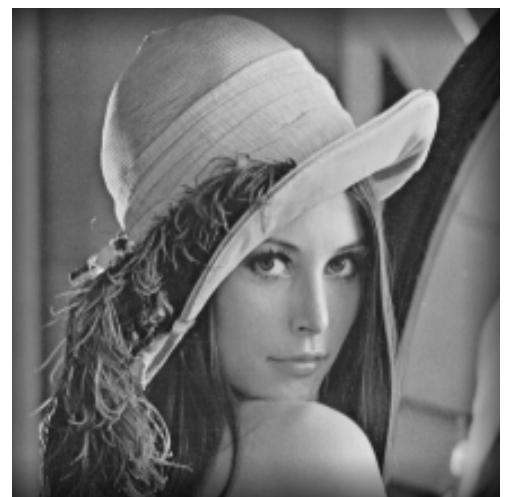

(a) Original Image

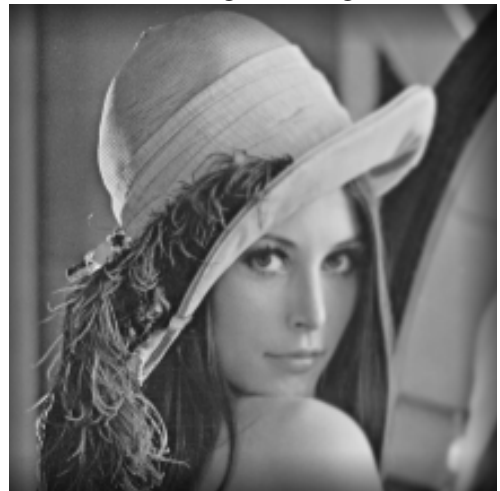

(b) Degraded Image $S N R=30.30$

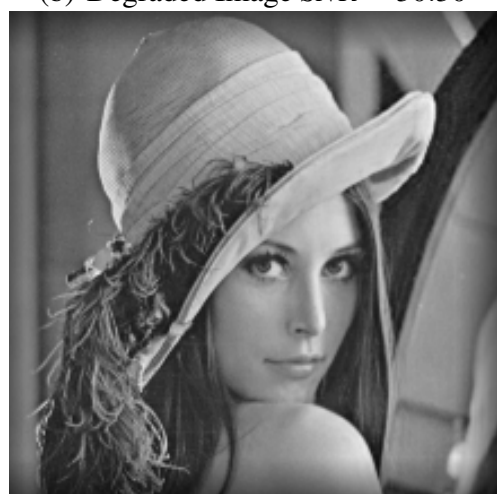

(c) Deblurred Image, $S N R=36.13$

FIGURE 15 - Restoration for $\sigma_{\eta}=0$.

\section{REFERENCES}

Beylkin, G., Coifman, R., and Rokhlin, V. (1991). Fast wavelet transform and numerical algorithm. Cотmun. Pure and Applied Math., $44: 141-183$.

Chambolle, A. and Pock, T. (2011). A first-order primaldual algorithm for convex problems with applications to imaging. Journal of Mathematical Imaging and Vision, 40(1) :120-145.

Chang, E.-C., Mallat, S., and Yap, C. (1999). Wavelet foveation. Applied and Computational Harmonic Analysis, 9:312-335.

Coifman, R. and Donoho, D. (1995). Translation-invariant 


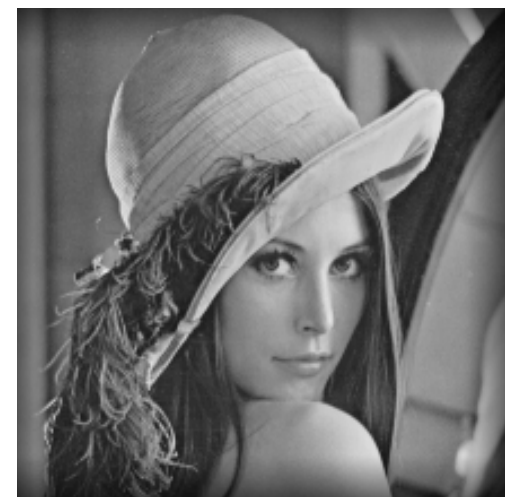

(a) Original Image

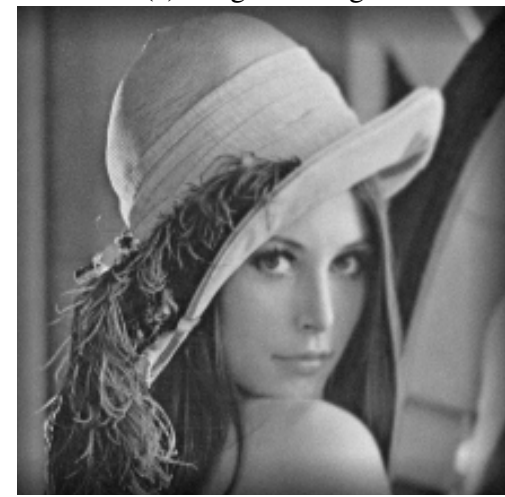

(b) Degraded Image $S N R=28.14$

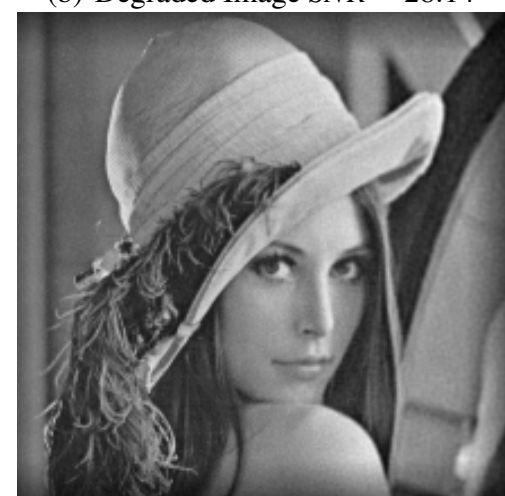

(c) Deblurred Image, $S N R=29.18$

FIGURE $16-$ Restoration for $\sigma_{\eta}=10^{-2}$.

de-noising. LECTURE NOTES IN STATISTICS-NEW YORK-SPRINGER VERLAG-, pages 125-125.

Coifman, R. and Meyer, Y. (1997). Wavelets, calderónzygmund and multilinear operators. Cambridge Studies in Advanced Math, 48.

Combettes, P., Wajs, V., et al. (2006). Signal recovery by proximal forward-backward splitting. Multiscale Modeling and Simulation, 4(4) :1168-1200.

Hansen, P., Nagy, J., and O'leary, D. (2006). Deblurring images : matrices, spectra, and filtering. Siam.

Kalifa, J., Mallat, S., and Rougé, B. (2003). Deconvolution by thresholdong in mirror wavelet bases. Image
Processing, IEEE Transactions on, 12(4) :446-457.

Kirshner, H., Sage, D., and Unser, M. (2011). 3D PSF models for fluorescence microscopy in ImageJ. In Proceedings of the Twelfth International Conference on Methods and Applications of Fluorescence Spectroscopy, Imaging and Probes (MAF'11), page 154, Strasbourg, France.

Malgouyres, F. (2002). A framework for image deblurring using wavelet packet bases. Applied and Computational Harmonic Analysis, 12(3) :309-331.

Nagy, J. and O'Leary, D. (1998). Restoring images degraded by spatially variant blur. SIAM Journal on Scientific Computing, $19: 1063$

Nemirovskii, A. and Yudin, D. (1979). Complexity of problems and efficiency of optimization methods.

Preibisch, S., Saalfeld, S., Schindelin, J., and Tomancak, P. (2010). Software for bead-based registration of selective plane illumination microscopy data. Nature methods, 7(6) :418-419.

Temerinac-Ott, M., Ronneberger, O., Ochs, P., Driever, W., Brox, T., and Burkhardt, H. (2011). Multiview deblurring for 3-d images from light sheet based fluorescence microscopy. Image Processing, IEEE Transactions on, (99) :1-1.

Wahba, G. (1990). Spline models for observational data, volume 59. Society for Industrial Mathematics.

Zhang, B., Zerubia, J., and Olivo-Marin, J. (2007). Gaussian approximations of fluorescence microscope point-spread function models. Applied Optics, 46(10) : 1819-1829. 